\title{
CAPACIDAD ANTIOXIDANTE in vitro Y EFECTO HIPOGLUCEMIANTE DE LA MACA NEGRA (Lepidium meyenii) PREPARADA TRADICIONALMENTE
}

\author{
Jhon Troya-Santos ${ }^{1 *}$, Neptalí Ale-Borja², Silvia Suárez-Cunza ${ }^{1}$
}

\begin{abstract}
RESUMEN
La maca (Lepidium meyenii) es una planta peruana nativa de los Andes centrales a la que se le atribuyen diversas propiedades, como mejorar la función sexual y la espermatogénesis, mejorar el aprendizaje, y la reducción del estrés; las mismas que podrían estar relacionadas con la variedad de este hipocótilo. Sin embargo, estas propiedades no sólo están basadas en el ecotipo o el color sino también en el tipo de prácticas del procesamiento de la maca. Tradicionalmente, la maca es preparada haciéndose hervir en agua. El objetivo del estudio fue medir la capacidad antioxidante in vitro de la maca negra de acuerdo a la forma de preparación tradicional y su efecto hipoglucemiante in vivo. Se hicieron tres preparados de la misma muestra sometidos a diferentes tiempos de cocción: 30, 45 y 60 minutos. A cada uno de los preparados se les determinó la capacidad antioxidante in vitro mediante las técnicas con 1,1-difenil-2-picril-hidrazilo (DPPH) y ácido 2,2'-azino-bis-3-etilbenzotiazolin6-sulfónico (ABTS). Para el estudio in vivo, se realizó la inducción de diabetes experimental con estreptozotocina y se les suplementó con maca diariamente, se evaluó la evolución glucémica en un periodo de 14 días. La maca a 60 minutos de cocción presentó mayor capacidad antioxidante $(6,8 \mu \mathrm{g} / \mathrm{mL}$ y $203,0 \mu \mathrm{g} / \mathrm{mL})$ mediante los reactivos DPPH y ABTS, respetivamente. La misma que mostró mantener los niveles glucémicos reducidos. La preparación de la maca de forma tradicional con 60 minutos de cocción obtiene un incremento de la capacidad antioxidante in vitro respecto a 30 y 45 minutos de cocción, además este tiene un efecto hipoglucemiante en un modelo de diabetes inducida con estreptozotocina.
\end{abstract}

Palabras clave: Maca, antioxidante, ensayos antioxidantes, hipoglucemiante.

\section{In vitro ANTIOXIDANT CAPACITY OF TRADITIONAL PREPARED BLACK MACA (Lepidium meyenii)}

\begin{abstract}
Maca is a Peruvian plant native to the central Andes attributed with sexual function, spermatogenesis, improve learning, memory and stress properties, which are reflective of the different colored types. However, the properties of maca are not only related to the

${ }^{1}$ Centro de Investigación de Bioquímica y Nutrición, Facultad de Medicina. UNMSM. Av. Grau 755. Lima 1, Perú

*jktroya@gmail.com

${ }^{2}$ Facultad de Química e Ingeniería Química. UNMSM.
\end{abstract}


ecotype or color, but also to its processing practices. Traditionally, maca is prepared by boiling. The objective of this study is to measure the in vitro antioxidant capacity of the black maca compared to the traditional preparation method and the maca hypoglycemic effect. The maca was divided in three groups based on the boiling time (30, 45 and 60 minutes). DPPH (2,2-diphenyl-1- picrylhydrazyl) and ABTS [2,2'-azinobis-(3-ethylbenzothiazoline)6-sulfonic acid] assays where performed in each of the maca sample in order to determine the total antioxidant capacity. A group of rats induced with diabetes via a streptozotocin shot and dairy were supplemented with maca, and the glycemic evolution was measured throughout a 14-day period. The maca boiled for 60 minutes presented the highest in vitro antioxidant capacity $(6,8 \mu \mathrm{g} / \mathrm{mL}$ y $203,0 \mu \mathrm{g} / \mathrm{mL})$ using the DPPH and ABTS assay, respectively. Streptozotocin induced diabetic rats supplemented with maca showed reduced glycemic level as well. Maca traditionally prepared for 60 minutes indicated higher in vitro antioxidant capacity compared to 30 and 45 minutes and had in vivo hypoglycemic capacity in a diabetes model induced by streptozotocin.

Key words: Maca, antioxidant, antioxidant essays, hypoglycemic.

\section{INTRODUCCIÓN}

La maca (Lepidium meyenii Walpers o Lepidium peruvianum Chacón) forma parte de la familia Brassicaceae, es una planta nativa de los Andes Centrales que crece dentro de los 4000-4500 msnm. Se le atribuyen varios beneficios como de mejorar la función sexual, la espermatogénesis, mejorar el aprendizaje, la memoria, el estrés y ser una efectiva solución a la fatiga incrementando la resistencia física e incluso tienen efectos como la de mejorar la fertilidad femenina y masculina ${ }^{1,2}$.

Se han descrito hasta trece variedades de maca de acuerdo al color del hipocótilo, que van del color blanco al negro ${ }^{3}$, la diferencia del color está asociada a las diversas concentraciones de los metabolitos bioactivos aun cuando estas variedades de maca contienen los mismos metabolitos secundarios. Por ejemplo, la variedad de maca negra presenta mayor concentración de glucosinolatos comparado a las variedades roja y amarilla ${ }^{4}$. Sin embargo, esta diferencia de las concentraciones de los metabolitos no sólo es producto del ecotipo de maca, sino también del tipo de prácticas del procesamiento de la misma posteriormente a la cosecha. Esparza et al. ${ }^{5}$, en el 2015, reportaron que existe un metabolismo endógeno de los isotiocianatos generados de la hidrólisis de los glucosinolatos durante el secado de la maca.

La maca está constituida por metabolitos primarios que corresponden a los componentes nutricionales. Sin embargo, también se han identificado metabolitos secundarios como compuestos fenólicos, a los cuales se les atribuye las propiedades biológicas y medicinales de la maca; entre estos, los macaenos, macamidas, glucosinalatos y alcaloides de maca que sólo se encuentran en la maca (lepidilina A, lepidilina B y el derivado de dehidropiridiina llamado macaridina $)^{6,7}$. 
La diabetes es una enfermedad compleja, de condición heterogénea que tiene como principal causa la disfunción de las células beta del páncreas, esencial para el mantenimiento de la homeostasis de la glucosa. Muchos factores influyen en la función de las células beta, entre ellos la hiperglicemia, la glucotoxicidad, la lipotoxicidad, la autoinmunidad, la inflamación, las adipoquinas, las incretinas, la resistencia a la insulina, e incluso el estrés oxidativo; estos pueden conllevar a un alto riesgo de complicaciones ${ }^{8}$. Se cree que el estrés oxidativo juega un papel muy importante en el desarrollo de complicaciones vasculares, las variaciones en los niveles de enzimas hacen a los tejidos susceptibles al estrés oxidativo; de acuerdo a estudios epidemiológicos, la mortalidad diabética puede explicarse notablemente por un incremento en las enfermedades vasculares derivados de la hiperglucemia ${ }^{9}$.

En los tratamientos de la diabetes se incluyen fármacos hipoglucemiantes orales e insulina, así como la modificación de los estilos de vida. Sin embargo, actualmente también se ha incluido terapias alternativas y complementarias, como el uso de diversas plantas medicinales mediadas por sus metabolitos secundarios ${ }^{10,11,12}$. Por ello, la maca, podría constituir potencialmente en un recurso de utilidad terapéutica, para contribuir a la calidad de vida de estos pacientes, sea por su propiedad antioxidante o por su propiedad hipoglucemiante.

El objetivo del presente estudio fue medir la capacidad antioxidante in vitro de la maca negra de acuerdo al tiempo de cocción en la forma tradicional de preparación y su acción hipoglucemiante in vivo en un modelo de diabetes experimental en ratas macho.

\section{PARTE EXPERIMENTAL}

El estudio se desarrolló en los laboratorios del Centro de Investigación de Bioquímica y Nutrición de la Facultad de Medicina de la UNMSM. Se usó los hipocótilos de la maca negra peruana cultivados en Carhuamayo, Junín a $4000 \mathrm{msnm}$. El extracto acuoso fue preparado de acuerdo al método usado tradicionalmente. Los hipocótilos de maca fueron cortados y secados en estufa a $37{ }^{\circ} \mathrm{C}$ para luego ser pulverizado con molino manual. Se colocó 40 gramos de maca pulverizada en un matraz con $200 \mathrm{~mL}$ de agua dejándose a hervir por tres tiempos diferentes. Así, se le denominó Maca A al hervido por 30 minutos, Maca B al de 45 minutos y Maca $\mathrm{C}$ al de 60 minutos. Los preparados fueron enfriados, filtrados y luego centrifugados a 2500 r.p.m por 5 minutos a $4{ }^{\circ} \mathrm{C}$ en una centrífuga Sorvall, rotor SS-34. Se recuperó el sobrenadante en tubos de vidrio y se almacenó a una temperatura de $8{ }^{\circ} \mathrm{C}$ para los análisis respectivos.

Se determinó la masa soluble de maca en cada preparado de las soluciones acuosas mediante gravimetría. Se pesó un mililitro de cada extracto de maca de cada tiempo de cocción, en un recipiente de peso conocido, y se dejó secar por un lapso de cinco días en la estufa a $37^{\circ} \mathrm{C}$, hasta peso constante, posteriormente se volvió a pesar los recipientes obteniendo el peso seco de la maca. 


\section{Reactivos}

Ácido 2,2'-azinobis (3 -etilbenzotiazolín) - 6- sulfónico (ABTS), 1,1-difenil-2-picrilhidrazilo $\left(\mathrm{DPPH}^{\bullet}\right.$ ) y compuesto ácido 6- hidroxi-2,5,7,8-tetrametilcromo-2-carboxílico (trolox) fueron adquiridos de Sigma - Aldrich.

\section{Capacidad antioxidante total}

Para esta evaluación de la maca se utilizaron dos metodologías: el ensayo con el radical libre catiónico 2,2'-azinobis (3 -etilbenzotiazolín) - 6- sulfónico (ABTS. ${ }^{+}$) y el ensayo con el radical libre 1,1-difenil-2-picrilhidrazilo (DPPH).

\section{Ensayo con el radical libre $\mathrm{ABTS}^{{ }^{+}}$}

Según la metodología desarrollada por Re et al. ${ }^{13}$ (1999) y modificada por Kuskoski et al. ${ }^{14}$ (2004), el radical ABTS ${ }^{++}$se obtuvo tras la reacción del compuesto ABTS (7 mM) con persulfato de potasio $(2,45 \mathrm{mM}$, concentración final $)$ incubados a temperatura ambiente $\left(25^{\circ} \mathrm{C}\right)$ y en oscuridad durante $16 \mathrm{~h}$. Una vez formado el radical $\mathrm{ABTS}^{\cdot+}$ se diluyó en agua bidestilada hasta obtener un valor de absorbancia de $0,70( \pm 0,02)$ a $754 \mathrm{~nm}$, esta es la absorbancia inicial. El tubo de reacción de $1 \mathrm{~mL}$ con $980 \mu \mathrm{L}$ del radical libre, contenía $20 \mu \mathrm{L}$ de la muestra de maca diluida con agua capaz de producir la disminución de la absorbancia del radical ABTS ${ }^{+}$ en el rango del 20 al 80\% de su absorbancia inicial después de siete minutos de incubación a temperatura ambiente alejado de la luz. El antioxidante sintético de referencia, trolox, se ensayó a una concentración de $0-15 \mu \mathrm{M}$ (concentración final). Los resultados se expresaron como capacidad antioxidante equivalente al trolox (TEAC-ABTS en $\mu \mathrm{mol}$ de Trolox/g de muestra) y en la concentración inhibitoria del 50\% (IC50). Se analizaron cada uno de los extractos a distintas concentraciones.

\section{Ensayo con el radical libre DPPH}

Se realizó según el método desarrollado por Brand-Williams et al. ${ }^{15}$ (1995), con modificaciones descritas por Kim et al. ${ }^{16}$ (2002). Se midió la absorbancia del radical DPPH $100 \mu \mathrm{M}(0,8 \mathrm{~mL})$ disuelto en metanol al $80 \%$. Se añade $0,4 \mathrm{~mL}$ de la muestra o patrón, se mezcla, y se mantiene en la oscuridad durante 30 minutos a temperatura ambiente. Las medidas se leen a $517 \mathrm{~nm}$. Los resultados se expresaron en la actividad equivalente a trolox (TEAC-DPPH en $\mu \mathrm{mol} / \mathrm{g}$ de muestra) previa preparación de una curva de calibración a concentraciones de 0,08-1,28 $\mathrm{mM}$ en disolución de metanol al $80 \%$. Esta evaluación se realizó para cada tiempo de hervor de la maca y luego se determinó la concentración inhibitoria del 50\% $\left(\mathrm{IC}_{50}\right)$.

\section{Actividad hipoglucemiante}

Se empleó ocho ratas macho de 4 meses de edad $(202,4+31,6 \mathrm{~g})$, de la raza Holtzman obtenidas del Instituto Nacional de Salud (Lima, Perú). Para el tratamiento de los animales se respetó las normas éticas para el tratamiento de animales de laboratorio, según la guía de manejo y cuidado de animales de laboratorio del Instituto Nacional de Salud (INS). Todos los grupos recibieron el concentrado comercial para animales de laboratorio Rodentina Purina ${ }^{\circledR}$ con $10 \%$ de la composición en grasa. Después del periodo de aclimatación de siete días, los animales se separaron al azar en dos grupos: STZ+Agua (Diabéticas y no alimentadas con maca) y STZ+Maca (Diabéticas y alimentadas con maca). Los animales se mantuvieron a 
una temperatura ambiente (aproximadamente $23^{\circ} \mathrm{C}+2$ ) con un ciclo luz/oscuridad 12:12 horas en el bioterio de la Facultad de Medicina de la UNMSM.

Para la inducción a diabetes se les suministró vía intraperitoneal una sola inyección de estreptozotocina $35 \mathrm{mg} / \mathrm{kg}$ peso en tampon citrato $10 \mathrm{mM}$ a pH 7,4. Después de cinco días de haber sido inducidas a la diabetes, se les administró el preparado de maca con mayor actividad antioxidante durante un periodo de 14 días en el grupo correspondiente, para ello se usó una sonda nasogástrica $\mathrm{N}^{\circ} 18$, suministrándole un volumen de $2 \mathrm{~mL}$ de solución diaria. La dosis de maca administrada diariamente a cada rata fue de $2 \mathrm{~g} \mathrm{~kg}^{-1}$ de peso del animal, el cual es equiparable con el consumo humano en los Andes del Perú ${ }^{17}$. Se realizó mediciones periódicas de las concentraciones de glucosa basal postprandial en las muestras de sangre de la vena de la cola de cada uno de los animales mediante el método conductivimétrico empleando el glucómetro Assure II.

Para el análisis estadístico se usó Shapiro Wilk para el análisis de la normalidad de las variables y los grupos dentro de las variables, y se evaluó la varianza aplicando el test de Levegne. La prueba paramétrica por el análisis ANOVA de comparaciones múltiples con post Hoc de Bonferroni. Se asumió un grado de significancia $($ alfa $=0,05)$.

\section{RESULTADOS Y DISCUSIÓN}

\section{Peso seco de extractos de maca}

Los tres tiempos de cocción para la maca presentan pesos secos (masa soluble) similares, sólo en el caso del grupo B se encontró un valor ligeramente por encima de $1 \mathrm{~g} / \mathrm{mL},(1,022$ $\mathrm{g} / \mathrm{mL})$.

Tabla 1. Peso seco de maca en cada tiempo de cocción en $\mathrm{g} / \mathrm{mL}$

\begin{tabular}{cl}
\hline MACA & Media $(\mathbf{g} / \mathbf{m L}) \pm$ DS \\
\hline A & $0,868 \pm 0,03$ \\
B & $1,022 \pm 0,16$ \\
C & $0,899 \pm 0,01$
\end{tabular}

Medida por triplicado. DS = Desviación estándar .

\section{Capacidad antioxidante total}

Los resultados de la capacidad antioxidante total de los tres extractos acuosos expresados como IC50 mostraron que la Maca C, que corresponde a una cocción de 60 minutos, tuvo el mejor comportamiento con ambas técnicas. En la tabla 2 los valores del IC50 empleando el radical libre $\mathrm{ABTS}^{\bullet+}$ varían entre $347,2 \mu \mathrm{g} / \mathrm{mL}$ y $203,0 \mu \mathrm{g} / \mathrm{mL}$; el menor valor significa una mayor capacidad antioxidante y corresponde a la Maca C, con 203,0 $\mu \mathrm{g} / \mathrm{mL}$. Con esta técnica la duplicación del tiempo mejora en un $41,5 \%$ el valor del $\mathrm{IC}_{50}$. La literatura refiere que 
la maca contiene además de glucosinolatos otros metabolitos secundarios tipo polifenoles especialmente catequinas que podrían estar explicando esta actividad antioxidante ${ }^{18}$. Los polifenoles por ser excelentes dadores de electrones o hidrógenos con la formación de radicales intermedios relativamente estables es encontrado en los compuestos que contienen capacidad antioxidante; además de estar relacionado con la capacidad de quelar metales. Sin embargo, los alcaloides, especialmente macamidas y macaenas, que son los principales constituyentes funcionales de la maca, también podrían estar relacionados con esta propiedad ya que se les ha relacionado con el tratamiento o prevención de la osteoporosis, cáncer de próstata y enfermedades del riñón, o su función potencial en el tratamiento de enfermedades cardiovasculares $^{19}$.

Tabla 2. $\mathrm{IC}_{50}$ por el ensayo $\mathrm{ABTS}^{\bullet+}$ para cada tiempo de cocción del extracto acuoso de maca.

\begin{tabular}{|c|c|c|c|}
\hline \multicolumn{5}{|c|}{ IC50 $(\boldsymbol{\mu g} / \mathbf{m L})$} \\
\hline MACA & Media & DS & CV \% \\
A & 347,2 & 28,1 & 8,09 \\
\hline B & 266,0 & 14,2 & 5,35 \\
\hline C & 203,0 & 17,2 & 8,46 \\
\hline Trolox & 154,8 & 4,1 & 2,63 \\
\hline
\end{tabular}

Medida por triplicado. (Media \pm Desviación estándar), CV: coeficiente de variación

En los ensayos de los tres grupos frente al DPPH (tabla 3) también la Maca C exhibió el mejor comportamiento antioxidante.

Tabla 3. $\mathrm{IC}_{50}$ por el ensayo DPPH para cada tiempo de cocción del extracto acuoso de maca.

\begin{tabular}{|c|lc|c|}
\hline \multicolumn{4}{|c|}{ IC $_{\mathbf{5 0}}(\boldsymbol{\mu g} / \mathbf{m L})$} \\
\hline MACA & Media & DS & CV \% \\
\hline A & 8,40 & 0,65 & 7,7 \\
\hline B & $8,50^{\mathbf{c}}$ & 0,58 & 6,9 \\
\hline C & $6,80^{\text {a,b }}$ & 0,34 & 4,9 \\
\hline Trolox & 4,70 & 0,04 & 0,8 \\
\hline
\end{tabular}

Medida por triplicado, (Media \pm Desviación estándar) ANOVA de comparaciones múltiples con post Hoc de Bonferroni, $\alpha=0,05$. a $=$ Diferencia estadística entre el grupo de 60 minutos y 30 minutos $(p=0,029), \mathrm{b}=$ Diferencia estadística entre el grupo 60 minutos y 45 minutos $(P=0,029), \mathrm{c}=$ Diferencia estadística entre el grupo 30 minutos y 45 minutos $(p=1,00)$. 
Los $\mathrm{IC}_{50}$ varían entre $6,80 \mu \mathrm{g} / \mathrm{mL}$ y $8,50 \mu \mathrm{g} / \mathrm{mL}$, la maca del grupo C presenta menor $\mathrm{IC}_{50}$ entre todos los tiempos de cocción $(6,8 \mu \mathrm{g} / \mathrm{mL})(p=0,0029)$, correspondiendo a una disminución de $19 \%$ al duplicar el tiempo de cocción. Estos resultados muestran que la Maca C, la que corresponde a una cocción de 60 minutos, es la que exhibe mejor capacidad antioxidante mediante las dos técnicas con los radicales libres $\mathrm{ABTS}^{\bullet+}$ y DPPH. El tiempo de cocción influye en la capacidad antioxidante de la maca probablemente porque favorece mecanismos endógenos que permiten la liberación de metabolitos secundarios solubles tipo polifenoles que pudieran estar confinados dentro de macromoléculas (proteínas o carbohidratos), esto en base a que trabajos previos en la literatura han demostrado que una serie de reacciones sólo toman lugar a través de acciones endógenas, procesos enzimáticos o no enzimáticos ${ }^{5}$.

\section{Determinación de equivalentes trolox (TEAC)}

Las curvas de calibración con los dos radicales libres para el estándar trolox produjeron gráficas lineales, en el ensayo con $\mathrm{ABTS}^{\bullet+}$ con un coeficiente de correlación $\mathrm{R}^{2}$ : 0,97221 y para el ensayo con DPPH un coeficiente de correlación de $\mathrm{R}^{2}$ : 0,99982. A partir de las cuales fue posible expresar la actividad antioxidante de los extractos acuosos de la maca evaluadas en $\mu \mathrm{g}$ trolox/g de extracto. En la tabla 4 se muestra la actividad antioxidante de los extractos acuosos de maca, obtenidos mediante los ensayos de DPPH y ABTS ${ }^{\bullet+}$ expresada en equivalentes trolox. Igualmente se identifica que el tiempo de cocción de 60 minutos de la Maca $\mathrm{C}$ es la que presentó mejor capacidad antioxidante.

Tabla 4. Equivalente Trolox para los ensayos $\mathrm{ABTS}^{\bullet+} \mathrm{y}_{\mathrm{DPPH}}^{\bullet}$. De los extractos acuosos de maca

\begin{tabular}{|c|l|l|}
\hline & \multicolumn{2}{|c|}{ TEAC ( $\mu$ g trolox /g de extracto) } \\
\hline MACA & ABTS $^{\bullet+}$ & DPPH \\
\hline A & 445,73 & 541,81 \\
\hline B & 581,81 & 537,35 \\
\hline C & 762,47 & 672,09 \\
\hline
\end{tabular}

Se han reportado criterios de selección para extractos vegetales con base en el $\mathrm{IC}_{50}$ evaluado frente a DPPH; considerando de alto potencial antioxidante aquellos con valores menores a $30 \mu \mathrm{g} / \mathrm{mL}$, con moderado potencial ubicados en un rango entre $30 \mu \mathrm{g} / \mathrm{mL}$ y $100 \mu \mathrm{g} / \mathrm{mL}$ y de bajo potencial antioxidante aquellos con un $\mathrm{IC}_{50}$ por encima de $100 \mu \mathrm{g} / \mathrm{mL}^{20}$. En nuestro trabajo la capacidad antioxidante de los extractos acuosos frente al radical DPPH medida en IC50, es mayor en la maca de 60 minutos de cocción, con $6,80 \mu \mathrm{g} / \mathrm{mL}$. Considerando nuestros resultados, la maca en los tres tiempos de cocción presenta un alto potencial antioxidante, sin embargo, la cocción por 60 minutos favorece en obtener el mayor potencial antioxidante. Los valores obtenidos de $\mathrm{IC}_{50}$ de la maca negra son menores a los reportados por Sandoval et al. ${ }^{21}$ (2002), donde encontraron un $\mathrm{IC}_{50}$ de $610 \mu \mathrm{g} / \mathrm{mL}$. Incluso es menor a otros ecotipos evaluados posteriormente por Carrión et al. ${ }^{22}$ (2009) con $\mathrm{IC}_{50}$ de DPPH para maca amarilla, blanca, morado: 367,$7 ; 317,5 ; 313 ; 4(\mu \mathrm{g} / \mathrm{mL})$, respectivamente. El valor obtenido es también 
menor a lo recientemente reportado por Doroteo et al. ${ }^{23}$ (2013) obteniendo resultados de $\mathrm{IC}_{50}$ mayor a $100 \mu \mathrm{g} / \mathrm{mL}$ en maca. Esta diferencia podría explicarse por la diferencia metodológica en la preparación del extracto de maca, además que existe una diferencia entre los trabajos respecto a la determinación del valor real de la concentración de la maca en los extractos evaluados. En el presente trabajo se consideró como la concentración de la maca al peso seco de la solución suministrada y no a la maca pulverizada utilizada para preparar la solución acuosa. Otra diferencia es el tiempo de cocción de 10 minutos empleados en los trabajos de Sandoval et al. ${ }^{21} \mathrm{y}$ de Carrión et al. ${ }^{22}$

La mayor capacidad antioxidante en la maca $\mathrm{C}$, cocción por 60 minutos expresado como $\mathrm{IC}_{50}$ en ambos ensayos ABTS ${ }^{\bullet+}$ y DPPH, demuestra que hay una alta correlación entre ambos ensayos (DPPH y $\mathrm{ABTS}^{\bullet+}$ ) para la maca, tal como lo observado en otras investigaciones ${ }^{24,25}$. Tanto para el ensayo $\mathrm{ABTS}^{\bullet+}$ al igual que para el ensayo DPPH, el tiempo de cocción de maca por 60 minutos también presentó la mejor capacidad antioxidante equivalente al trolox. En otros trabajos, como el de Becerra et al. ${ }^{26}, 2011$, se ha visto una subestimación del valor de equivalente trolox en DPPH comparado al método con $\mathrm{ABTS}^{+}$, en el presente trabajo no se ha podido encontrar este tipo de subestimaciones. Lo cual nos indica que ambos métodos son comparables y pueden ser usados. A pesar que se ha encontrado una alta correlación entre ambos ensayos (DPPH y $\mathrm{ABTS}^{\bullet+}$ ), tal como se ha observado en otras investigaciones el ABTS presenta importantes ventajas, como de tener amplia solubilidad ${ }^{24,25}$, permitiendo ensayos con extractos o moléculas tanto de naturaleza lipofílica como hidrofílica, además del menor tiempo para la evaluación.

\section{Glucemia en ratas diabéticas}

En los grupos diabéticos, con suplemento y sin suplemento de maca de 60 minutos de cocción (maca $\mathrm{C}$ ), se observó que el primer día que se indujo a diabetes ambos grupos presentaron una similar concentración de glucosa. En el quinto día el grupo STZ+Agua incrementó en $136,1 \%$ la glucemia mientras que el grupo STZ+Maca se incrementó en 75,1\% la glucemia. El pico de glicemia en el grupo STZ+Agua se alcanzó en el día 9 mientras que el pico de glicemia en el grupo STZ+Maca se alcanzó en el día 14, es decir que el tratamiento retrasó el pico de glicemia. Las ratas diabéticas que recibieron maca mantuvieron un grado de glucemia por debajo de los $300 \mathrm{mg} / \mathrm{mL}$ en el pico más alto, es decir alcanzó un incremento máximo de $107,0 \%$ con respecto al día de inicio con estreptozotocina, no se halló diferencia significativa durante todo el tiempo de tratamiento (tabla 5). Sin embargo, en aquellas ratas diabéticas sin suplemento de maca se apreció que la glucemia promedio alcanzó un incremento de $272,4 \%$, mayor al doble del grupo que recibió dieta con maca, encontrándose diferencia significativa $(p=0,04)$, entre todas las medias del tiempo de evaluación (tabla 5). 
Tabla 5. Evolución de la glucemia en las ratas diabéticas tratadas con extracto de Maca $\mathrm{C}$

\begin{tabular}{|l|c|c|c|c|c|}
\hline & \multicolumn{5}{|c|}{ DÍA (Glucosa mg/mL) } \\
\hline TRATAMIENTO & 1 & 5 & 9 & 14 & 19 \\
\hline STZ+Agua 1(n=4) $^{\text {a }}$ & 115,8 & 273,3 & 431,0 & 306,5 & 225,0 \\
\hline${\text { STZ+Maca C }(n=4)^{b}}^{\text {b }}$ & 117,3 & 205,3 & 232,8 & 242,8 & 186,8 \\
\hline
\end{tabular}

Prueba no paramétrica de Friedman, $\mathrm{a}=$ Hay diferencia entre las medidas repetidas en el grupo STZ + Agua $(p=0,027)$ $X^{2}=11.0, \mathrm{~b}=$ No hay diferencia entre las medidas repetidas entre los grupos STZ+Maca $(p=0,92) X^{2}=8 . \alpha=0,05$ para ambos grupos evaluados.

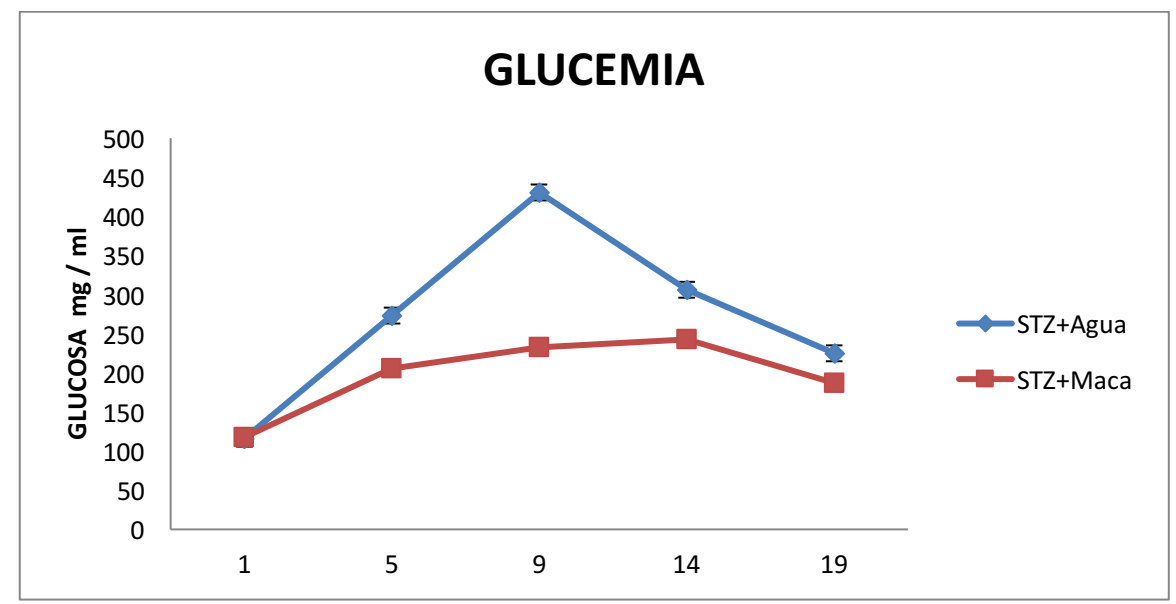

Figura 1. Evolución glucémica de los tratamientos con y sin maca. Se realizó el suplemento de maca desde el primer día de evaluación y determinación de diabetes.

Del resultado de la evaluación de la glucemia de los grupos tratados con maca se observó que la maca permitió mantener grados moderados de glucemia (figura 1). Rodrigo et al. ${ }^{10}$, en el 2010, también llegaron a la conclusión que la maca amarilla tiene la capacidad de mejorar el metabolismo de la glucosa reduciendo en 50\% la glucemia, es decir tiene un efecto hipoglucemiante. En los resultados obtenidos desde el pico más alto de glucemia, la maca sólo pudo lograr una reducción en $23 \%$ de la glucemia, esto se explica por la diferencia de tiempo de tratamiento en ambos trabajos, 46 días en el trabajo de Rodrigo et $a l .{ }^{10}, 26$ días más de tratamiento que en este trabajo; esta reducción de la glucemia podría estar explicada debido a que la maca incrementó la producción de la insulina en un $22 \%$ por mecanismos todavía desconocidos ${ }^{10}$. En el 2013, Gonzales et al. ${ }^{27}$ también reportaron un efecto hipoglucemiante de la maca negra en un modelo de diabetes experimental con 
estreptozotocina en ratones. Esta propiedad podría estar explicada en el trabajo reciente de Yin et al. ${ }^{19}$ en el 2016, donde encontraron que algunos componentes de la maca, alcaloides y macaínas, tienen una actividad inhibitoria de enzima convertidora de angiotensina I, pudiendo funcionar potenciando la reducción del azúcar en la sangre como los antidiabéticos orales e insulina, aunque sus mecanismos aún se desconocen.

\section{CONCLUSIONES}

La preparación de la maca de forma tradicional con un tiempo de cocción de 60 minutos produce un incremento de la capacidad antioxidante in vitro de 41,5\% y 19\% en los valores de IC50 empleando $\mathrm{ABTS}^{\bullet+}$ y DPPH, respectivamente, frente al tiempo de cocción de 30 minutos. Evaluado in vivo, en ratas diabéticas inducidas con estreptozotocina y tratadas con maca negra se observa que la hiperglicemia alcanza un incremento de $107 \%$ frente al $272 \%$ en el grupo de ratas diabéticas sin tratamiento con maca negra, lo que refleja su potencial hipoglicemiante.

\section{AGRADECIMIENTOS}

El presente trabajo tuvo el apoyo económico parcial de la Facultad de Medicina de la UNMSM. Un agradecimiento especial a la Mg. Inés Arnao por su apoyo en el laboratorio "Marino Villavicencio" del CIBN y a Rudy López por su apoyo técnico.

\section{REFERENCIAS BIBIOGRÁFICAS}

1. Gonzales GF, Gasco M, Cordova A, Chung A, Rubio J, Villegas L. Effect of Lepidium meyenii (Maca) on spermatogenesis in male rats acutely exposed to high altitude (4340 m). J Endocrinol. 2004; 180:87-95.

2. Sandoval M, Okuhamaa NN, Angelesa FM, Melchora V V, Condezob L A, Laob J, Millera M JS. Antioxidant activity of the cruciferous vegetable Maca (Lepidium meyenii). Food Chem. 2002; 79: 207-213.

3. Tello J, Hermann M, Calderón A. La maca (Lepidium meyenii Walp.) cultivo alimenticio potencial para las zonas altoandinas. Bol Lima. 1992; 14:59-66.

4. Meissner H O, Mscisz A, Mrozikiewicz M, Baraniak M, Mielcarek S, Kedzia B, Piatkowska E, Jólkowska J, Pisulewski P. Peruvian Maca (Lepidium peruvianum): (I) Phytochemical and Genetic Diferences in three Maca Phenotypes. Int J Biomed Sci. 2015; 2(3): 131-145.

5. Esparza E, Hadzich A, Kofer W, Mithöfer A, Cosio EG. Bioactive maca (Lepidium meyenii) alkamides are a result of traditional Andean postharvest drying practices. Phytochem. 2015;116: 138-148

6. Castaño MP. Maca (Lepidium peruvianum Chacon): composición química y propiedades farmacológicas. Rev Fitoterapia. 2008; 8(1):23-30. 
7. Dini I, TenoreG.C, Dini A. Glucosilates from Maca (Lepidium Meyenni). Biochem System Ecol. 2002; 30: 1087-1090.

8. Cernea S y Dobreanu M. Diabetes and beta cell function: from mechanisms to evaluation and clinical implications. Biochem Med (Zagreb). 2013; 23(3): 266-280.

9. Turan B. Role of antioxidants in redox regulation of diabetic cardiovascular complications. Curr Pharm Biotechnol. 2010 ;11(8):819-36.

10. Rodrigo ME, Valdivieso R, Suaréz S, Oriondo R, Oré R I. Disminución del daño oxidativo y efecto hipoglicemiante de la maca (Lepidium meyenii Walp) en ratas con diabetes inducida por streptozotocina. An Fac Med. 2010; 71 Supl 1.

11. Oré S. Efectos hipolipémico y antioxidante de Lepidium meyenii Walp en ratas. [Tesis Doctoral en Ciencias Biológicas]. Lima: Universidad Nacional Mayor de San Marcos; 2008 .

12. Giovannini P, Howes MJ R and Edwards SE. Medicinal plants used in the traditional management of diabetes and its sequelae in Central America: A review. J Ethnopharmacol. 2016; 26 (184):58-71.

13. Re R, Pellegrini Ni, Proteggente A, Pannala A, Yang M, Rice-Evans C. Antioxidant activity applying an improved ABTS radical cation decolorization assay. Free Radic Biol Med. 1999; 26: 1231-1237.

14. Kuskoski E M, Asueroro A G, Troncoso A M, Garcia-Parilla M C, Fett R. Actividad antioxidante de pigmentos antocianicos. Rev Bras Ciênc Tecnol Alim. 2004; 24 (4): 691-693.

15. Brand-Williams W, Cuvelier ME, Berset C. Use of a free radical method to evaluate antioxidant activity. LWT Food Sci Technol. 1995; 28, 25-30.

16. Kim D-O, Lee KW, Lee H J, Lee C Y. Vitamin C equivalent antioxidant capacity (VCEAC) of phenolic phytochemicals. J Agric Food Chem. 2002; 50: 3713-3717.

17. Valerio LG, Jr, Gonzales GF (2005). Toxicological aspects of the South American herbs cat's claw (Uncaria tomentosa) and Maca (Lepidium meyenii): a critical synopsis. Toxicol Rev. 2005; 24:11-35

18. Gonzales GF, Villaorduña L, Gasco M, Rubio J, Gonzales C. Maca (Lepidium meyenii Walp), una revisión sobre sus propiedades biológicas. Rev Peru Med Exp Salud Publica. 2014; 31(1):100-10.

19. Yi F, Tan XL, Yan X y Liu HB. In silico profiling for secondary metabolites from Lepidium meyenii (maca) by the pharmacophore and ligand-shape-based joint approach. Chin Med. 2016; 11: 42.

20. Ramos A, Vizoso A, Piloto J, García A, Rodriguez CA, Rivero R. Screening and antimutagenicity via antioxidant activity in Cuban medicinal plants. J Ethnopharmacol. 2003; 87: 241-246.

21. Sandoval M, Okuhamaa NN, Angelesa FM, Melchora V V, Condezob L A, Laob J, Millera M JS. Antioxidant activity of the cruciferous vegetable Maca (Lepidium meyenii). Food Chem. 2002; 79: 207-213.

22. Carrión J, León K, Santiago J. Actividad antioxidante de tres ecotipos de maca (Lepidium Mayenii Walp) tratada con radiación gamma. Rev Per Quím Ing Quím. 2009. 12 (2), 72 77.

23. Doroteo VH, Diaz C, Terry C, Rojas R. Compuestos fenólicos y actividad antixoidante 
in vitro de 6 plantas peruanas. Rev Soc Quím Perú. 2013; 79 (1): 13-20.

24. Babbar N, Oberoi HS, Uppal DS, Patil RT. Total phenolic content and antioxidant capacity of extracts obtained from six important fruit residues. Food Res Int. 2011; 44: 391-396.

25. Floegel A, Kim D, Chung S, Koo S I, Chun O K. Comparison of ABTS/DPPH assays to measure antioxidant capacity in popular antioxidant-rich US foods. J Food Comp Anal. 2011; 24: 1043-1048.

26. Becerra M, Machado P, Campos A, Do Prado G, Carvalho C, Arraes G, Gomes T. Bioactive compounds and antioxidant activity of fresh exotic fruits from northeastern Brazil. Food Res Int. 2011; 44: 2155-2159

27. Gonzales GF, Gonzales-Castañeda C y Gasco M. A mixture of extracts from Peruvian plants (black maca and yacon) improves sperm count and reduced glycemia in mice with streptozotocin-induced diabetes. Toxicol Mech Methods. 2013; 23(7): 509-518. 